\title{
Avaliação do risco de quedas dos idosos residentes em instituição de longa permanência no Brasil: uma revisão integrativa
}

\author{
Verônica Porto de Freitas*, Naiara Andrade do Carmo*, Brenda de Couto Andreotti Raimundo ** \\ Ariana Oliveira Santos ${ }^{* * *}$, Claudinéia Matos de Araújo ${ }^{* * * *}$, Luciana Araújo dos Reis ${ }^{* * * * *}$
}

\section{Resumo}

As quedas entre idosos são um dos principais problemas clínicos e de saúde pública, devido à sua alta incidência, às complicações e aos altos custos assistenciais. Nesta perspectiva, o presente estudo teve por objetivo analisar a avaliação do risco de quedas em idosos residentes em instituição de longa permanência a partir de uma revisão integrativa. Foram analisados artigos cadastrados nas bases de pesquisa SciELO e Bireme, no período de 2010 a 2015, em língua portuguesa e resultantes do cruzamento dos seguintes descritores: instituição de longa permanência, idosos e risco de quedas, utilizando-se o indicador boleano (AND). Dos 19 artigos utilizados, evidenciaram-se institucionalização, sexo feminino, idade superior a 70 anos, presença de comorbidades, uso de medicamentos e polifarmácia, cômodos e período mais comum, como determinantes para tornar o idoso vulnerável a episódios de queda.

Palavras-chave: Avaliação. Idoso. Quedas.

\section{Introdução}

O envelhecimento faz parte do processo biológico e natural da vida. Nesse processo, os indivíduos apresentam deterioração e declínio biológicos e psicológicos, dependentes das relações das dimensões cognitivas e psicoafetivas, o que interfere na personalidade, no afeto e no comportamento. Apesar da

* Fisioterapia da Universidade Estadual do Sudoeste da Bahia. Bolsista de iniciação científica FAPESB. E-mail: verinha.porto.freitas@gmail.com

** Acadêmica do curso de Fisioterapia da Universidade Estadual do Sudoeste da Bahia. Bolsista de iniciação científica CNPq. E-mail: naiarauesb4@gmail.com

**** Acadêmica do curso de Fisioterapia da Universidade Estadual do Sudoeste da Bahia. Bolsista de iniciação científica FAPESB. E-mail: brendaandreotti@outlook.com

***** Acadêmica do curso de Fisioterapia da Universidade Estadual do Sudoeste da Bahia. Bolsista de iniciação científica UESB. E-mail: hariianna_oliveira@hotmail.com

***** Graduada em Fisioterapia, mestre em Enfermagem e Saúde pela Universidade Estadual do Sudoeste da Bahia. Docente assistente do curso de Fisioterapia na mesma instituição. E-mail: neialis@hotmail.com

****** Graduada em Fisioterapia, mestre e doutora em Ciências da Saúde pela Universidade Federal do Rio Grande do Norte. Pós-doutoramento em Saúde Coletiva/ISC-UFBA. Docente da Universidade Estadual do Sudoeste da Bahia. Endereço para correspondência: Av. José Moreira Sobrinho, sem número, Jequiezinho. Jequié, BA. CEP: 45200-000. E-mail: lucianauesb@yahoo.com.br

$\rightarrow$ http://dx.doi.org/10.5335/rbceh.v14i3.6201

Recebido em: 17.07.2016. Aceito em: 14.03.2018. 
predeterminação da idade cronológica, são grandes as diferenças individuais e dependentes do meio em que o idoso vive, tais como estilo de vida, condições socioeconômicas e doenças crônicas, o que pode levar a formas completamente diferentes de envelhecimento em indivíduos de mesma idade cronológica (FECHINE; TROMPIERI, 2015).

O envelhecimento populacional é uma realidade em toda a humanidade como uma consequência do processo de desenvolvimento econômico. A transição demográfica que tem ocorrido no Brasil nas últimas 7 décadas, decorrente da redução da taxa de fecundidade e de natalidade, da inserção crescente da mulher no mercado de trabalho, das mudanças na nupcialidade e da queda na taxa de mortalidade inversamente proporcional a taxa de morbidade da população idosa (adultos de idade igual ou superior a 60 anos), resulta em idosos que se encontram com morbidades crônicas e sem condições físicas, emocionais, sociais, econômicas e familiares que lhes permitam conviver em comunidade (CAMARANO; KANSO, 2010).

Dentre as diversas alterações estruturais ocorridas nos idosos, uma das consequências mais evidentes são as quedas. Pois a estabilidade corporal depende de uma integração adequada entre os receptores de informações de componentes sensoriais, cognitivos, integrativos centrais e musculoesqueléticos. As quedas parecem resultar do efeito cumulativo de alterações relacionadas a idade, doenças e meio ambiente inadequado. Assim, os fatores de risco são classificados em: intrínsecos
- histórico de quedas, idade, sexo, uso de medicamentos, condição clínica, distúrbio de marcha e equilíbrio, sedentarismo, etc. -, extrínsecos - iluminação inadequada, superfícies escorregadias, tapetes soltos ou com dobras, ausência de corrimão, etc. - e comportamentais, que dependem do grau de exposição ao risco - os mais inativos, possivelmente pela fragilidade, e os mais ativos, pelo maior grau de exposição aos fatores extrínsecos (BUKSMAN et al., 2008).

Segundo o Relatório Mundial de Envelhecimento e saúde da Organização Mundial da Saúde (OMS, 2015), dois terços da população mundial são idosos (pessoas com idade acima de 65 anos), e estima-se que poderá chegar a $75 \% \mathrm{em}$ 2025. Já, no Brasil, 8,17\% da população é idosa (pessoas com 60 anos ou mais de idade), podendo chegar a $11,30 \%$ em 2025, segundo dados do Instituto Brasileiro de Geografia e Estatística (IBGE) (2016). Cerca de 28\% dos idosos brasileiros caem ao menos uma vez ao ano, e $13 \%$, de forma recorrente, o que decorre em morbimortalidade, dependência e redução da autonomia e da qualidade de vida (FERREIRA; YOSHITOME, 2010). A projeção de quedas para 2050 é de que, aproximadamente, um a cada três idosos sofrerá uma ou mais quedas por ano (ANTES et al., 2013).

A queda e o medo de cair são consideradas síndromes comuns em idosos, com resultados potencialmente graves, como redução da confiança na capacidade de caminhar, depressão, sentimentos de desamparo, isolamento social e alterações comportamentais que refletem na sua 
mobilidade funcional (ANTES et al., 2013). Dessa forma, as consequências das quedas são não apenas físicas, mas também psicológicas e sociais, tais como hospitalização, institucionalização ou reabilitação, levando a uma redução da autonomia deste idoso, bem como ao aumento da sua dependência e dos custos para a saúde pública (ÁLVARES; LIMA; SILVA, 2010).

Quando se trata de queda, a incidência de lesões é superior nos idosos institucionalizados (ANTES et al., 2013), comparativamente aos idosos não institucionalizados, o que torna o acidente uma fonte importante de morbidade e mortalidade nos idosos institucionalizados (ANTES et al., 2013). Estima-se que as quedas ocasionem de 6 a 9 mil hospitalizações de idosos institucionalizados, com uma média de 12 a 20 dias de hospitalização (ÁLVARES; LIMA; SILVA, 2010).

Dessa forma, o objetivo do presente estudo é avaliar o risco de quedas dos idosos residentes em instituição de longa permanência (Ilpi) a partir de uma revisão integrativa da literatura.

\section{Metodologia}

Para o alcance do objetivo proposto, optou-se por realizar uma revisão integrativa, qualitativa e descritiva a partir de artigos científicos publicados sobre o risco de queda em idosos residentes em uma Ilpi. Esse tipo de revisão é definido como um método capaz de sintetizar os resultados oriundos de pesquisas sobre um determinado tema ou questão, de forma sistemática e ordenada, com o intuito de contribuir com o tema ou a questão abordado(a) (ROMAN; FRIEDLANDER, 1998).

Como critérios de inclusão de artigos, foram considerados os artigos cadastrados nas bases de pesquisa SciELO e Bireme, do período de 2010 a 2015 e em língua portuguesa e resultantes do cruzamento dos seguintes descritores: instituição de longa permanência, idosos e risco de quedas. E como critérios de exclusão, foram desconsiderado artigos que não abordavam o tema, que só disponibilizavam o resumo e que eram de revisão.

Após o levantamento das publicações, os resumos foram lidos e analisados segundo os critérios de inclusão e exclusão preestabelecidos. Fez-se uma triagem quanto à relevância dos estudos e à propriedade com que respondiam ao objetivo deste estudo, chegando a uma amostra final de 19 artigos, que foram lidos e analisados na íntegra. Para a apuração dos dados, elaborou-se um instrumento com as seguintes variáveis: autores, tipo de estudo, amostra, local do estudo, ano de publicação, principais resultados e conclusões. A análise dos artigos procedeu-se de modo descritivo, e os resultados foram apresentados em quadros, divididos nas seguintes categorias analíticas: principais resultados encontrados sobre a prevalência de quedas em idosos residentes em Ilpis, principais resultados encontrados sobre os riscos intrínsecos para quedas de residentes em Ilpis, principais resultados encontrados quanto aos riscos extrínsecos para quedas de residentes em Ilpis e principais resultados encontrados 
quanto às consequências das quedas em idosos residentes em Ilpis.

A coleta de dados foi realizada em dezembro de 2015 e janeiro de 2016 . Do cruzamento (instituição de longa permanência AND idosos AND risco de queda/ idoso AND risco de queda/instituição de longa permanência AND idoso), foram encontrados 488 artigos; desses, foram pré-selecionados 24 por meio da análise do título, do resumo e da metodologia. Foram excluídos 5 artigos por não atenderem aos critérios de inclusão e exclusão. Por fim, chegou-se aos 19 artigos utilizados no presente estudo, conforme ilustra o seguinte fluxograma.

Figura 1 - Fluxograma de seleção dos artigos com descritores e indicador boleano utilizados

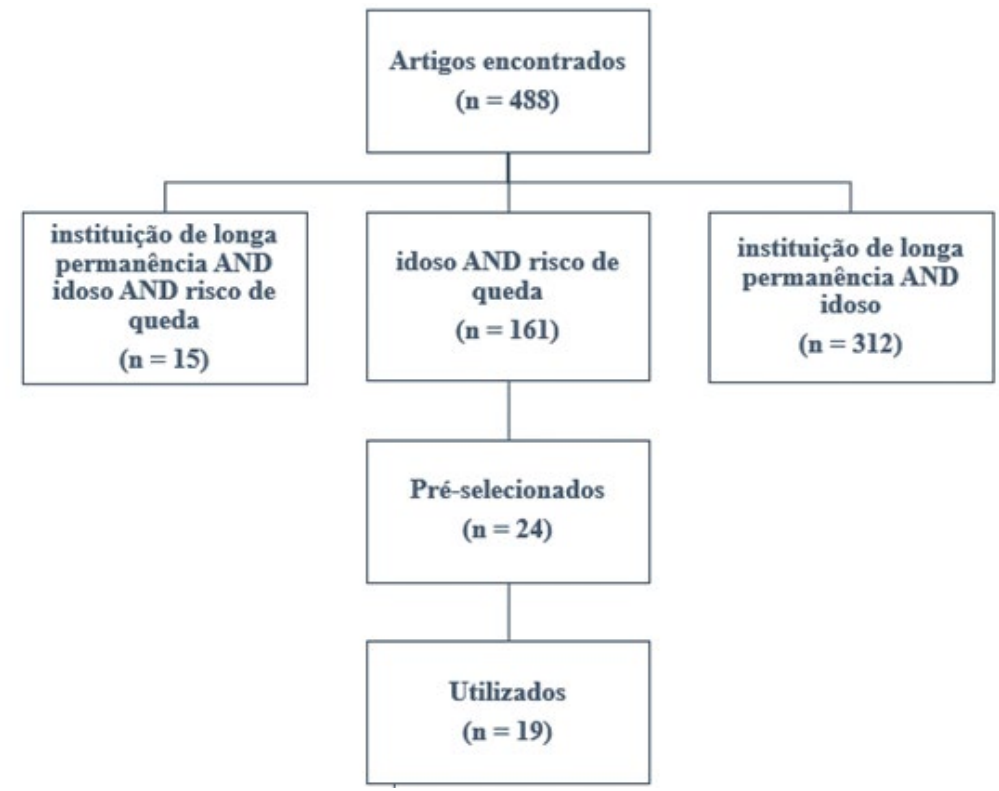

Legenda: $\mathrm{n}$ = número de artigos utilizados em cada sessão.

Fonte: elaboração dos autores.

\section{Resultados e discussão}

\section{Caracterização dos estudos}

Os artigos apresentam diferenças quanto ao tipo de estudo, sendo 13 $(68,42 \%)$ com delineamento transversal, $4(21,05 \%)$ descritivos e exploratórios e $2(10,53 \%)$ prospectivos. Dos desenhos analíticos, observacionais, exploratórios, são estudos que buscam uma maior compreensão dos fatores de risco para queda de idosos institucionalizados, bem como as medidas preventivas mais adequadas. Todos os artigos foram publicados em língua portuguesa, e os estudos, realizados em Ilpis públicas ou filantrópicas, com apenas um estudo em Ilpi particular, mas todas destinadas a idosos com renda baixa. 
Quadro 1 - Caracterização da produção científica quanto a autores, tipo de estudo, amostragem, local, periódico e ano de publicação

\begin{tabular}{|c|c|c|c|c|c|}
\hline Autores & Tipo de Estudo & Amostra & Local & Periódico & Ano \\
\hline Almeida e Neves & Transversal & $\begin{array}{l}113 \text { idosos } \\
\text { institucionalizados }\end{array}$ & IIpi & $\begin{array}{l}\text { Kairós: } \\
\text { gerontologia }\end{array}$ & 2012 \\
\hline $\begin{array}{l}\text { Álvares, Lima e } \\
\text { Silva }\end{array}$ & Transversal & $\begin{array}{l}243 \text { idosos } \\
\text { institucionalizados }\end{array}$ & 19 Ilpis de Pelotas, RS & $\begin{array}{l}\text { Cad. Saúde } \\
\text { Pública }\end{array}$ & 2010 \\
\hline $\begin{array}{l}\text { Alves e } \\
\text { Scheicher }\end{array}$ & Transversal & $\begin{array}{l}45 \text { idosos } \\
\text { institucionalizados } \\
\text { e } 43 \text { não } \\
\text { institucionalizados }\end{array}$ & Garça, SP & $\begin{array}{l}\text { Rev. Bras. } \\
\text { Geriatr. Gerontol. }\end{array}$ & 2011 \\
\hline Borges et al. & Transversal & $\begin{array}{l}54 \text { idosos } \\
\text { institucionalizados }\end{array}$ & Ilpi de Fortaleza, CE & $\begin{array}{l}\text { Acta Paul } \\
\text { Enferm. }\end{array}$ & 2013 \\
\hline $\begin{array}{l}\text { Reis, Rocha e } \\
\text { Duarte }\end{array}$ & $\begin{array}{l}\text { Analítico com } \\
\text { delineamento transversal } \\
\text { e abordagem quantitativa }\end{array}$ & $\begin{array}{l}62 \text { idosos } \\
\text { institucionalizados }\end{array}$ & $\begin{array}{l}\text { Ilpis de Vitória da } \\
\text { Conquista, BA, e Jequié, BA }\end{array}$ & $\begin{array}{l}\text { Revista Baiana } \\
\text { de enfermagem }\end{array}$ & 2014 \\
\hline $\begin{array}{l}\text { De Souza } \\
\text { Teixeira et al. }\end{array}$ & Transversal & $\begin{array}{l}14 \text { idosos } \\
\text { institucionalizados }\end{array}$ & Ilpi de Santa Maria, RS & $\begin{array}{l}\text { Kairós: } \\
\text { gerontologia }\end{array}$ & 2014 \\
\hline $\begin{array}{l}\text { Del Duca, Antes } \\
\text { e Hallal }\end{array}$ & $\begin{array}{l}\text { Epidemiológico de caráter } \\
\text { transversal e exploratório }\end{array}$ & $\begin{array}{l}466 \text { idosos } \\
\text { institucionalizados }\end{array}$ & $\begin{array}{l}25 \text { llpis da zona urbana de } \\
\text { Pelotas, RS }\end{array}$ & $\begin{array}{l}\text { Rev. Bras. } \\
\text { Epidemiol. }\end{array}$ & 2013 \\
\hline $\begin{array}{l}\text { Dos Santos } \\
\text { Lopes, Passerini } \\
\text { e Travensolo }\end{array}$ & Prospectivo de coorte & $\begin{array}{l}7 \text { idosos } \\
\text { institucionalizados }\end{array}$ & - & $\begin{array}{l}\text { Semina: Ciências } \\
\text { Biológicas e da } \\
\text { Saúde }\end{array}$ & 2010 \\
\hline Ferreira et al. & $\begin{array}{l}\text { Transversal com } \\
\text { delineamento quantitativo }\end{array}$ & $\begin{array}{l}20 \text { idosos } \\
\text { institucionalizados }\end{array}$ & $\begin{array}{l}\text { Ilpi privada de São José } \\
\text { do Rio Preto, SP }\end{array}$ & $\begin{array}{l}\text { ConScientiae } \\
\text { Saúde }\end{array}$ & $2013 a$ \\
\hline Ferreira et al. & $\begin{array}{l}\text { Transversal com } \\
\text { delineamento quantitativo }\end{array}$ & $\begin{array}{l}20 \text { idosos } \\
\text { institucionalizados }\end{array}$ & $\begin{array}{l}\text { Ilpi privada de São José } \\
\text { do Rio Preto, SP }\end{array}$ & $\begin{array}{l}\text { Kairós: } \\
\text { gerontologia }\end{array}$ & $2013 b$ \\
\hline Gonçalves et al. & Transversal & $\begin{array}{l}10 \text { idosos } \\
\text { institucionalizados }\end{array}$ & $\begin{array}{l}\text { Ilpi particular de } \\
\text { Uruguaiana, RS }\end{array}$ & $\begin{array}{l}\text { Estud. } \\
\text { Interdiscipl. } \\
\text { Envelheci. }\end{array}$ & 2014 \\
\hline Lojudice et al. & $\begin{array}{l}\text { Descritvo de corte } \\
\text { transversal }\end{array}$ & $\begin{array}{l}105 \text { idosos } \\
\text { institucionalizados }\end{array}$ & $\begin{array}{l}\text { Instituições azilares: } \\
\text { Associação São Vicente } \\
\text { de Paulo, Recanto } \\
\text { Monsenhor Albino, } \\
\text { Recanto Nosso Lar e } \\
\text { Sociedade Espírita Boa } \\
\text { Nova, Catanduva, SP }\end{array}$ & $\begin{array}{l}\text { Rev. Bras. } \\
\text { Geriatr. Gerontol. }\end{array}$ & 2010 \\
\hline $\begin{array}{l}\text { Menezes e } \\
\text { Bachion }\end{array}$ & $\begin{array}{l}\text { Descritivo-exploratório } \\
\text { com abordagem } \\
\text { quantitativa }\end{array}$ & $\begin{array}{l}59 \text { idosos } \\
\text { institucionalizados }\end{array}$ & 5 Ilpis de Goiânia, GO & Rev. Eletr. Enf. & $2012 a$ \\
\hline $\begin{array}{l}\text { Menezes e } \\
\text { Bachion }\end{array}$ & Prospectivo & $\begin{array}{l}59 \text { idosos } \\
\text { institucionalizados }\end{array}$ & Ilpis de Goiás & $\begin{array}{l}\text { Ver. Bras. } \\
\text { Oftalmol. }\end{array}$ & $2012 b$ \\
\hline Silva et al. & $\begin{array}{l}\text { Descritivo, observacional } \\
\text { com característica } \\
\text { transversal direta e de } \\
\text { natureza quantitativa }\end{array}$ & $\begin{array}{l}47 \text { idosos } \\
\text { institucionalizados }\end{array}$ & Ilpi de Teresina, PI & $\begin{array}{l}\text { Ver. Bras. } \\
\text { Geriatr. Gerontol. }\end{array}$ & 2013 \\
\hline Souza et al. & $\begin{array}{l}\text { Descritivo, exploratório, } \\
\text { de natureza quantitativa }\end{array}$ & $\begin{array}{l}50 \text { idosos } \\
\text { institucionalizados }\end{array}$ & Ilpi de Belo Horizonte, MG & $\begin{array}{l}\text { R. Enferm. Cent. } \\
\text { O. Min. }\end{array}$ & 2012 \\
\hline Uchida e Borges & $\begin{array}{l}\text { Descritivo, observacional } \\
\text { do tipo transversal }\end{array}$ & $\begin{array}{l}27 \text { idosos } \\
\text { institucionalizados }\end{array}$ & Ilpi de Santos, SP & $\begin{array}{l}\text { Kairós: } \\
\text { gerontologia }\end{array}$ & 2013 \\
\hline Valcarenghi et al. & $\begin{array}{l}\text { Quantitativo, do tipo } \\
\text { exploratório e descritivo }\end{array}$ & $\begin{array}{l}30 \text { idosos } \\
\text { institucionalizados }\end{array}$ & $\begin{array}{l}\text { Ilpi do Rio Grande do Sul } \\
\text { (Brasil) }\end{array}$ & $\begin{array}{l}\text { Acta. Paul. } \\
\text { Enferm. }\end{array}$ & 2011 \\
\hline Valcarenghi et al. & $\begin{array}{l}\text { Quantitativo, exploratório, } \\
\text { descritivo e observacional }\end{array}$ & $\begin{array}{l}30 \text { idosos } \\
\text { institucionalizados }\end{array}$ & $\begin{array}{l}\text { Ilpi do Rio Grande do Sul } \\
\text { (Brasil) }\end{array}$ & Rev. Rene. & 2014 \\
\hline
\end{tabular}

Fonte: elaboração dos autores. 


\section{Principais resultados encontrados sobre a prevalência de quedas em idosos residentes em Ilpis}

Alves e Scheicher (2011), em concordância com Reis, Rocha e Duarte (2014), apontam que a própria institucionalização representa um fator de risco para episódios de quedas de idosos, por viverem em isolamento social e por serem menos ativos, quando comparados a idosos não institucionalizados, uma vez que a inatividade acelera o processo de envelhecimento e o surgimento de complicações, com perda progressiva da funcionalidade.

Da mesma forma, Uchida e Borges (2013) ressaltam que a institucionalização desenvolve no idoso um perfil clinicofuncional e psicocognitivo mais associado aos fatores de risco para quedas, como redução da capacidade funcional, maior dependência, estado depressivo, déficit cognitivo e medo de cair. Para Lopes, Passerini e Travensolo (2010), ocorre também a perda da autonomia, tornando o idoso mais frágil, uma vez que a independência funcional está sujeita a recrutamento motor, equilíbrio, resistência cardiovascular e motivação, fatores comprometidos na população em questão.

Segundo Valcarenghi et al. (2011) e Reis, Rocha e Duarte (2014), os idosos recém chegados às Ilpis apresentam maior risco de queda por não estarem acostumados à estrutura física do novo ambiente. Del Duca, Antes e Hallal (2013) observam ainda que tanto o sofrimento gerado pela ausência de familiares como um ambiente pobre, inseguro e em condições clínicas ruins aumentam as chances de sofrer quedas.

Quadro 2 - Principais resultados encontrados em estudos que contemplaram a categoria prevalência de quedas em idosos residentes em Ilpis

\begin{tabular}{|c|c|c|}
\hline Autores & Título & Principais resultados \\
\hline $\begin{array}{l}\text { Alves e } \\
\text { Scheicher }\end{array}$ & $\begin{array}{l}\text { Equilíbrio postural e risco } \\
\text { para queda em idosos da } \\
\text { cidade de Garça, SP }\end{array}$ & $\begin{array}{l}\text { Houve diferença entre os escores da Escala de Berg nas populações estudadas }(p< \\
0,0001) \text {. Os resultados mostraram que a população institucionalizada apresenta nove } \\
\text { vezes mais risco de cair do que a não institucionalizada ( } p=0,0001) \text {. }\end{array}$ \\
\hline $\begin{array}{l}\text { Reis, } \\
\text { Rocha e } \\
\text { Duarte }\end{array}$ & $\begin{array}{l}\text { Quedas: risco e fatores as- } \\
\text { sociados em idosos institu- } \\
\text { cionalizados }\end{array}$ & $\begin{array}{l}62,9 \% \text { dos idosos foram vítimas de quedas; as causas de maior distribuição foram: difi- } \\
\text { culdade de caminhar, alteração de equilíbrio e tontura/vertigem, todos com } 16,1 \% \text { de in- } \\
\text { cidência cada. Em relação ao risco, no teste de TUG, a queda foi classificada com baixo } \\
\text { risco; na escala de Berg, houve maior frequência de risco de quedas }(61,3 \%) \text {. }\end{array}$ \\
\hline $\begin{array}{l}\text { Uchida e } \\
\text { Borges }\end{array}$ & $\begin{array}{l}\text { Quedas em idosos institu- } \\
\text { cionalizados }\end{array}$ & $\begin{array}{l}\text { Observou-se que apenas seis idosos caíram nos últimos doze meses }(22,2 \%) \text {, tota- } \\
\text { lizando nove quedas. No entanto, dezesseis }(59,3 \%) \text { relataram algum medo de cair. } \\
\text { Todos os idosos participantes do estudo apresentavam de quatro a onze fatores de } \\
\text { risco para quedas, sendo a presença de doença crônica, da polifarmácia, o uso de } \\
\text { medicamento psicotrópico e o déficit cognitivo os fatores de risco modificáveis mais } \\
\text { frequentes. Já a idade elevada ( }>75 \text { anos) e o sexo feminino são os fatores que mais } \\
\text { se destacaram entre os não modificáveis. }\end{array}$ \\
\hline $\begin{array}{l}\text { Dos Santos } \\
\text { Lopes, } \\
\text { Passerini e } \\
\text { Travensolo } \\
\end{array}$ & $\begin{array}{l}\text { Eficácia de um protocolo } \\
\text { fisioterapêutico para equilí- } \\
\text { brio em idosos instituciona- } \\
\text { lizados }\end{array}$ & $\begin{array}{l}\text { O protocolo de tratamento adotado no estudo gerou melhora no equilíbrio do grupo } \\
\text { participante, minimizando os riscos de déficit de equilíbrio nos idosos. }\end{array}$ \\
\hline $\begin{array}{l}\text { Valcarenghi } \\
\text { et al. }\end{array}$ & $\begin{array}{l}\text { Alterações na funcionalida- } \\
\text { de/cognição e depressão } \\
\text { em idosos institucionaliza- } \\
\text { dos que sofreram quedas }\end{array}$ & $\begin{array}{l}\text { Verificou-se maior ocorrência de queda entre mulheres e na população com baixa es- } \\
\text { colaridade. O risco de quedas aumentou com a idade e nos idosos com menor tempo } \\
\text { de institucionalização. Identificou-se a influência do uso de medicamentos nas quedas. } \\
\text { Os idosos que caíram apresentaram independência para as atividades de vida diária } \\
\text { (AVDs). Dos } 20 \text { idosos com escore sugestivo para déficit cognitivo, } 10 \text { sofreram quedas. } \\
\text { Não houve relação significativa entre depressão e quedas nos investigados. }\end{array}$ \\
\hline $\begin{array}{l}\text { Del Duca, } \\
\text { Antes e } \\
\text { Hallal }\end{array}$ & $\begin{array}{l}\text { Quedas e fraturas entre resi- } \\
\text { dentes de instituições de lon- } \\
\text { ga permanência para idosos }\end{array}$ & $\begin{array}{l}\text { Ter idade avançada, apresentar incapacidade funcional em uma a cinco atividades } \\
\text { da vida diária, residir em instituições públicas/ filantrópicas e ter sido hospitalizado no } \\
\text { último ano associaram-se à ocorrência de queda. O alto índice de quedas e fraturas } \\
\text { entre residentes de llpis revela a fragilidade da população avaliada. }\end{array}$ \\
\hline
\end{tabular}

Fonte: elaboração dos autores. 


\section{Principais resultados encontrados sobre os riscos intrínsecos para quedas residentes em Ilpis}

Os estudos de Álvares, Lima e Silva (2010), Lojudice et al. (2010), De Souza Teixeira et al. (2014), Ferreira et al. (2013a, 2013b), Almeida e Neves (2012), Reis, Rocha e Duarte (2014) e Valcarenghi et al. (2014) evidenciam que $o$ sexo feminino sofre mais quedas do que o sexo masculino, tanto por mulheres viverem por mais tempo quanto por serem mais acometidas por fatores intrínsecos e extrínsecos.

Entre os fatores intrínsecos, destacam-se alterações hormonais devido à redução de estrógeno, que leva a uma maior prevalência de osteoporose e à maior perda de massa óssea, além de diminuir a qualidade e a força da massa muscular, causando maiores fragilidade e vulnerabilidade; mulheres também apresentam maior prevalência de doenças crônicas (ÁLVARES; LIMA; SILVA, 2010), como artrose, artrite, etc. (SILVA et al., 2013). Entre os fatores extrínsecos, estão inadequada ingestão nutricional, resultando em sarcopenia (SILVA et al., 2013), e maior envolvimento com as atividades domésticas (ÁLVARES; LIMA; SILVA, 2010).

Segundo Valcarenghi et al. (2014), a idade aumenta substancialmente o risco de queda, fator evidenciado também por Uchida e Borges (2013) e Ferreira et al. (2013a, 2013b), devido ao próprio processo de envelhecimento, no qual os idosos, muitas vezes, tornam-se menos ativos, com consequente redução da capacidade física, desenvolvendo incapacidades e ficando dependentes e vulneráveis à prevalência de doenças crônico-degenerativas não transmissíveis.

Borges et al. (2013) acrescentam ainda que, embora a presença de doenças crônicas não seja sempre acompanhada de fragilidade, seus efeitos nocivos cumulativos durante o envelhecimento aumentam a probabilidade de o idoso se tornar frágil em decorrência dos desfechos clínicos que possivelmente possa apresentar, sendo mais frequente o aumento da fragilidade com o avanço da idade, com maior prevalência em idosos com 80 anos de idade ou mais.

Conforme Valcarenghi et al. (2011), essa redução da capacidade física deve-se à perda da força muscular e de outras características físicas, como perda da massa muscular e diminuição da flexibilidade (MENEZES; BACHION, 2012a), redução da elasticidade, prejuízos da estabilidade e da dinâmica articular, alterações dos sistemas sensorial, vestibular e somatossensorial e nervosos, comprometendo os mecanismos de controle postural e implicando alterações de marcha, postura e equilíbrio (SOUZA et al., 2012; ALVES; SCHEICHER, 2011).

Em contrapartida, Lojudice et al. (2010) não perceberam relação entre queda e longevidade em seu estudo; e Del Duca, Antes e Hallal (2013) e Álvares, Lima e Silva (2010) defendem que idosos longevos (acima de 70 anos de idade) com várias incapacidades movimentam-se menos, realizam menos tarefas e, em alguns casos, encontram-se acamados, 
tornando-se a idade um fator protetor, uma vez que assim ficam menos expostos aos fatores de risco de queda numa instituição e recebem maior supervisão.

Idosos institucionalizados e acometidos por demências e depressão apresentam declínio funcional progressivo e perda de autonomia, distúrbios cognitivos e déficit de atenção (VALCARENGHI et al., 2014), contribuindo para a redução do desempenho em atividades físicas e o aumento da fraqueza muscular global, com prejuízo nas atividades de vida diária (FERREIRA et al., 2013a), aumentando sua vulnerabilidade a quedas. Apesar de Valcarenghi et al. (2011) não terem evidenciado influência signi- ficativa entre alterações na capacidade funcional, demência, depressão e queda, reconhecem que o déficit cognitivo aumenta o risco de quedas.

A doença de Alzheimer (DA) causa importante déficit no equilíbrio e dificuldade na marcha, aumentando o risco de quedas (FERREIRA et al., 2013b). Além do declínio cognitivo, diminuições nas capacidades visual (MENEZES; BACHION, 2012b) e espacial, auditivas e do equilíbrio, principalmente quando associadas a idade avançada, sexo feminino e fatores ambientais negativos, repercutem diretamente no aumento do risco de queda dessa população (GONÇALVES et al., 2014; LOJUDICE et al., 2010).

Quadro 3 - Principais resultados encontrados na categoria riscos intrínsecos para quedas em idosos residentes em Ilpis

(continua...)

\begin{tabular}{|c|c|c|}
\hline Autores & Título & Principais resultados \\
\hline $\begin{array}{l}\text { Álvares, Lima e } \\
\text { Silva }\end{array}$ & $\begin{array}{l}\text { Ocorrência de quedas em idosos re- } \\
\text { sidentes em instituições de longa per- } \\
\text { manência em Pelotas, Rio Grande do } \\
\text { Sul, Brasil }\end{array}$ & $\begin{array}{l}\text { Houve diferença entre os escores da escala de Berg nas populações } \\
\text { estudadas }(p<0,0001) \text {. Os resultados mostraram que a população } \\
\text { idosa institucionalizada apresenta nove vezes mais risco de cair do } \\
\text { que a população não institucionalizada }(p=0,0001) \text {. }\end{array}$ \\
\hline $\begin{array}{l}\text { De Souza } \\
\text { Teixeira et al. }\end{array}$ & $\begin{array}{l}\text { Prevalência do risco de quedas em } \\
\text { idosos de uma instituição de longa } \\
\text { permanência de Santa Maria (RS) }\end{array}$ & $\begin{array}{l}\text { Foi identificada alta prevalência do risco de quedas, principalmente } \\
\text { em idosas, quando comparadas aos idosos homens. }\end{array}$ \\
\hline Ferreira et al. & $\begin{array}{l}\text { Risco de queda em idosos com do- } \\
\text { ença de Alzheimer institucionalizados }\end{array}$ & $\begin{array}{l}\text { Verificou-se que a maioria dos participantes }(75 \%) \text { era do sexo fe- } \\
\text { minino, com idade média de } 77,8 \pm 10,3 \text { anos e tempo de institucio- } \\
\text { nalização de } 2,4 \pm 1,7 \text { anos. Foi encontrado alto risco de queda nos } \\
\text { portadores de DA ( } 70 \%) \text {, verificando-se que, quanto maior a idade, } \\
\text { maior é o risco. }\end{array}$ \\
\hline Ferreira et al. & $\begin{array}{l}\text { Risco de queda em idosos institucio- } \\
\text { nalizados com doença de Alzheimer }\end{array}$ & $\begin{array}{l}\text { Foram encontrados maiores escores para alto risco de queda nos } \\
\text { idosos portadores de DA }(70 \%) \text {, com um coeficiente de correlação ( } r \\
=0,9999) \text { extremamente significante }(p=0,0079) \text {. }\end{array}$ \\
\hline $\begin{array}{l}\text { Reis, Rocha e } \\
\text { Duarte }\end{array}$ & $\begin{array}{l}\text { Quedas: risco e fatores associados } \\
\text { em idosos institucionalizados }\end{array}$ & $\begin{array}{l}62,9 \% \text { dos idosos participantes foram vítima de quedas; as causas de } \\
\text { maior distribuição foram: dificuldade de caminhar, alteração de equilí- } \\
\text { brio e tontura/vertigem, todos com, respectivamente, } 16,1 \% \text {. Em rela- } \\
\text { ção ao risco, no teste de TUG, a queda foi classificada com baixo risco; } \\
\text { na escala de Berg, houve maior frequência }(61,3 \%) \text { de risco de quedas. }\end{array}$ \\
\hline $\begin{array}{l}\text { De Souza } \\
\text { Rocha e Duarte }\end{array}$ & $\begin{array}{l}\text { Prevalência do risco de quedas em } \\
\text { idosos de uma instituição de longa } \\
\text { permanência de Santa Maria (RS) }\end{array}$ & $\begin{array}{l}\text { Foi identificada alta prevalência do risco de quedas, principalmente } \\
\text { nas idosas, quando comparadas aos idosos homens. }\end{array}$ \\
\hline $\begin{array}{l}\text { Valcarenghi } \\
\text { et al. }\end{array}$ & $\begin{array}{l}\text { Ações institucionais alicerçadas em } \\
\text { diagnósticos de enfermagem para } \\
\text { prevenção de quedas em idosos }\end{array}$ & $\begin{array}{l}\text { Foram elaboradas propostas de ações institucionais relacionadas } \\
\text { a receptibilidade ao idoso pela instituição, adaptação do ambiente, } \\
\text { treinamento físico dos idosos, investigação e ensino de equipamen- } \\
\text { tos auxiliares, promoção da independência, avaliação das condições } \\
\text { clínicas e custo benefício de tratamento medicamentoso. }\end{array}$ \\
\hline
\end{tabular}




\begin{tabular}{|c|c|c|}
\hline Silva et al. & $\begin{array}{l}\text { Correlação entre o risco de queda e } \\
\text { autonomia funcional em idosos insti- } \\
\text { tucionalizados }\end{array}$ & $\begin{array}{l}47 \% \text { dos idosos apresentaram maior risco de queda, e } 53 \% \text {, menor } \\
\text { risco. Ser do sexo feminino aumenta } 3,9 \text { vezes as chances de queda } \\
\text { em relação ao sexo masculino. }\end{array}$ \\
\hline $\begin{array}{l}\text { Uchida e } \\
\text { Borges }\end{array}$ & Quedas em idosos institucionalizados & $\begin{array}{l}\text { Observou-se que a frequência de quedas na população do estudo foi } \\
\text { de apenas seis idosos, que caíram nos últimos doze meses }(22,2 \%) \text {, } \\
\text { totalizando nove quedas. No entanto, dezesseis }(59,3 \%) \text { relataram } \\
\text { algum medo de cair. Todos os idosos participantes do estudo apre- } \\
\text { sentam de quatro a onze fatores de risco para quedas, sendo a pre- } \\
\text { sença de doença crônica, a polifarmácia, o uso de medicamento psi- } \\
\text { cotrópico e o déficit cognitivo os fatores de risco modificáveis mais } \\
\text { frequentes. Já a idade elevada (> } 75 \text { anos) e o sexo feminino são os } \\
\text { fatores que mais se destacam, dentre os não modificáveis. }\end{array}$ \\
\hline Borges et al. & $\begin{array}{l}\text { Avaliação da fragilidade de idosos ins- } \\
\text { titucionalizados }\end{array}$ & $\begin{array}{l}\text { Houve correlações positivas entre fragilidade, sexo, idade, presença } \\
\text { de comorbidades, índice de massa corporal e necessidade e quan- } \\
\text { tidade de medicamentos. A fragilidade em idosos institucionalizados } \\
\text { sofre influência das características sociodemográficas e clínicas. }\end{array}$ \\
\hline $\begin{array}{l}\text { Valcarenghi } \\
\text { et al. }\end{array}$ & $\begin{array}{l}\text { Alterações na funcionalidade/cogni- } \\
\text { ção e depressão em idosos institucio- } \\
\text { nalizados que sofreram quedas }\end{array}$ & $\begin{array}{l}\text { Verificou-se que a maioria dos participantes eram mulheres e ti- } \\
\text { nham baixa escolaridade; o risco de quedas aumentou com a ida- } \\
\text { de; e idosos com menor tempo de institucionalização caíram mais. } \\
\text { Identificou-se a influência do uso de medicamentos nas quedas. Os } \\
\text { idosos que caíram apresentaram independência para as AVDs; dos } \\
20 \text { idosos com escore sugestivo para déficit cognitivo, } 10 \text { sofreram } \\
\text { quedas. Não houve influência significativa entre depressão e quedas } \\
\text { nos investigados. }\end{array}$ \\
\hline $\begin{array}{l}\text { Menezes e } \\
\text { Bachion }\end{array}$ & $\begin{array}{l}\text { Ocorrência de quedas e seu contexto } \\
\text { num seguimento de dois anos em ido- } \\
\text { sos institucionalizados }\end{array}$ & $\begin{array}{l}\text { O estudo apresentou } 54,2 \% \text { de prevalência de quedas. As quedas } \\
\text { ocorreram, predominantemente, durante o dia, fora do ambiente } \\
\text { interno institucional, durante a deambulação, em piso de cimento, } \\
\text { seco, a partir de tropeços em algo, quando estavam calçando chi- } \\
\text { nelos. }\end{array}$ \\
\hline Souza et al. & $\begin{array}{l}\text { Perfil de idosas que sofreram quedas } \\
\text { em uma instituição de longa perma- } \\
\text { nência }\end{array}$ & $\begin{array}{l}38 \% \text { das idosas investigadas sofreram quedas, com uma média de } \\
\text { idade de } 79,8 \text { anos. A maior ocorrência de eventos foi no quarto } \\
(38,9 \%) \text {, no pátio }(22,2 \%) \text { e no refeitório }(22,2 \%) \text {. Em relação às } \\
\text { consequências das quedas, } 72,2 \% \text { das idosas sofreram escoria- } \\
\text { ções, e } 5,6 \% \text {, fraturas. }\end{array}$ \\
\hline $\begin{array}{l}\text { Alves e } \\
\text { Scheicher }\end{array}$ & $\begin{array}{l}\text { Equilíbrio postural e risco para queda } \\
\text { em idosos da cidade de Garça, SP }\end{array}$ & $\begin{array}{l}\text { Houve diferença entre os escores da escala de Berg nas populações } \\
\text { estudadas }(p<0,0001) \text {. Os resultados mostraram que a população } \\
\text { institucionalizada apresenta nove vezes mais risco de cair do que } \\
\text { idosos não institucionalizados }(p=0,0001) \text {. }\end{array}$ \\
\hline Lojudice et al. & $\begin{array}{l}\text { Quedas de idosos institucionalizados: } \\
\text { ocorrência e fatores associados }\end{array}$ & $\begin{array}{l}40 \% \text { dos idosos relataram quedas nos últimos seis meses, e os fa- } \\
\text { tores de risco considerados significativos foram: sexo feminino, uso } \\
\text { de medicamentos, visão deficiente, ausência de atividade física, } \\
\text { presença de osteoartrose, depressão, déficit de força de preensão } \\
\text { palmar e distúrbios no equilíbrio e na marcha. }\end{array}$ \\
\hline $\begin{array}{l}\text { Del Duca, Antes } \\
\text { e Hallal }\end{array}$ & $\begin{array}{l}\text { Quedas e fraturas entre residentes } \\
\text { de instituições de longa permanência } \\
\text { para idosos }\end{array}$ & $\begin{array}{l}\text { Ter idade avançada, apresentar incapacidade funcional de uma a } \\
\text { cinco atividades da vida diária, residir em instituições públicas/fi- } \\
\text { lantrópicas e ter sido hospitalizado no último ano associaram-se à } \\
\text { ocorrência queda no último ano. O alto índice de quedas e fraturas } \\
\text { entre residentes de llpis revela a fragilidade da população avaliada. }\end{array}$ \\
\hline Gonçalves et al. & $\begin{array}{l}\text { Avaliação das funções cognitivas, } \\
\text { qualidade do sono, tempo de reação } \\
\text { e risco de quedas em idosos institu- } \\
\text { cionalizados }\end{array}$ & $\begin{array}{l}\text { A preocupação com a possibilidade de quedas na realização de ati- } \\
\text { vidades cotidianas em geral, avaliada por meio da EEQ, apresentou } \\
\text { média de } 31,75 \text { pontos, o que revela medo de cair. Acredita-se que } \\
\text { possa haver relação entre a preocupação com a possibilidade de } \\
\text { sofrer quedas e o declínio das atividades de interação social, de } \\
\text { prática de atividades físicas e de vida diária. }\end{array}$ \\
\hline
\end{tabular}

Fonte: elaboração dos autores. 


\section{Principais resultados encontrados quanto aos riscos extrínsecos para quedas em residentes em Ilpis}

Borges et al. (2013) perceberam um predomínio do sexo masculino na instituição por eles estudada, fator resultante de o local acolher, em sua maioria, idosos ex-moradores de rua, perdidos, abandonados, sem referências familiar e social.

Souza et al. (2012) e Lojudice et al. (2010) demonstram que há uma prevalência de quedas entre idosos que usam medicamentos psicoativos, ansiolíticos e antidepressivos, por consequência de seus efeitos, causando hipotensão postural, sedação, arritmias, tremores e fraquezas. É aumentado o risco na polifarmácia, situação em que o idoso utiliza diversos medicamentos ao mesmo tempo pra tratar doenças crônicas mais comuns, além da utilização de um fármaco para corrigir o efeito adverso do outro.

Conforme Borges et al. (2013), quanto mais frágil é o idoso, maior a quantidade de medicamento prescritos. Nessa perspectiva, Valcarenghi et al. (2011, 2014) evidenciaram a influência do uso de medicamentos na ocorrência de quedas, pois, mesmo sem informar a causa dessa influência, o uso de drogas - ansiolíticos, anti-hipertensivos, antidepressivos, diuréticos e tranquilizantes - aumenta o risco, especialmente em idosos mais frágeis, uma vez que geram alterações fisiológicas no processo do envelhecimento, vulnerabilizando o idoso à decorrência de adoecimentos.

Confirmando a influência do fator medicamentos, Reis, Rocha e Duarte (2014) ratificaram que boa parte dos idosos que sofreram quedas fazia uso de medicamen- tos, principalmente anti-hipertensivos, sendo este um fator de causa extrínseca para episódios de queda. Complementarmente, Uchida e Borges (2013) destacam em seu trabalho que os medicamentos podem causar, além da hipotensão postural, sedação excessiva, diminuição do tempo de reação e alteração de equilíbrio, favorecendo a ocorrência de quedas.

Quanto aos locais de maior ocorrência de quedas, o quarto prevalece, seguido do pátio; em relação à posição das quedas, o predomínio é em pé, seguido de levantar da cama, do sofá ou da cadeira (REIS; ROCHA; DUARTE, 2014) e na transferência de sentado para em pé, ou o inverso (ÁLVARES; LIMA; SILVA, 2010). Nesse aspecto, Souza et al. (2012) acrescentam como um dos locais de ocorrência de quedas o refeitório, e Álvares, Lima e Silva (2010), o banheiro.

Contrapondo-se a maioria das pesquisas, Lojudice et al. (2010) perceberam em seu trabalho que o banheiro é o local de maior ocorrência. Complementarmente Almeida e Neves (2012) reafirmam que o quarto é o local de maior relato de quedas, sendo que esse ambiente deveria corresponder a uma área de maior circulação, estruturado e organizado de forma a promover uma mobilidade individual, segura e limitadora do potencial de quedas.

Álvares, Lima e Silva (2010) reportam ainda que as quedas são consequências de escorregão, tontura, desequilíbrio, vertigem e causa acidental. Outros fatores desfavoráveis, como piso escorregadio, molhado ou irregular e calçados inadequados, são apontados por Menezes e Bachion (2012a). Reis, Rocha e Duarte (2014) citam vestuário inadequado e grande como fator que dificulta a marcha, levando o idoso a pisar na própria roupa e cair. 
Quanto ao período de maior ocorrência das quedas, conforme Álvares, Lima e Silva (2010), foi o turno diurno, contrapondo-se à hipótese de Menezes e Bachion (2012a) de que durante a noite o idoso tem maior risco de queda, ao levantar para beber água ou para ir ao banheiro, por micções frequentes ou incontinências (SOUZA et al., 2012), deparando-se com condições ambientais desfavoráveis, como a iluminação inadequada.

Quadro 4 - Principais resultados encontrados na categoria riscos extrínsecos para quedas em idosos residentes em Ilpis

\begin{tabular}{|c|c|c|}
\hline Autores & Título & Principais resultados \\
\hline $\begin{array}{l}\text { Del Duca, } \\
\text { Antes e } \\
\text { Hallal }\end{array}$ & $\begin{array}{l}\text { Quedas e fraturas entre resi- } \\
\text { dentes de instituições de longa } \\
\text { permanência para idosos }\end{array}$ & $\begin{array}{l}\text { Ter idade avançada, apresentar incapacidade funcional de uma a cinco atividades } \\
\text { da vida diária, residir em instituições públicas/filantrópicas e ter sido hospitalizado no } \\
\text { último ano associaram-se à ocorrência de queda no último ano. O índice de quedas } \\
\text { e fraturas entre residentes de Ilpis revela a fragilidade da população avaliada. }\end{array}$ \\
\hline Borges et al. & $\begin{array}{l}\text { Avaliação da fragilidade de ido- } \\
\text { sos institucionalizados }\end{array}$ & $\begin{array}{l}\text { Houve correlações positivas entre fragilidade, sexo, idade, presença de comorbi- } \\
\text { dades, índice de massa corporal e necessidade e quantidade de medicamentos. } \\
\text { A fragilidade em idosos institucionalizados sofre influência das características so- } \\
\text { ciodemográficas e clínicas. }\end{array}$ \\
\hline Souza et al. & $\begin{array}{l}\text { Perfil de idosas que sofreram } \\
\text { quedas em uma instituição de } \\
\text { longa permanência }\end{array}$ & $\begin{array}{l}38 \% \text { das idosas participantes sofreram quedas, com uma média de idade de } 79,8 \\
\text { anos. A maior ocorrência de eventos foi no quarto }(38,9 \%) \text {, no pátio }(22,2 \%) \text { e no } \\
\text { refeitório }(22,2 \%) \text {. Em relação às consequências das quedas, } 72,2 \% \text { das idosas } \\
\text { sofreram escoriações, e } 5,6 \% \text {, fraturas. }\end{array}$ \\
\hline $\begin{array}{l}\text { Lojudice } \\
\text { et al. }\end{array}$ & $\begin{array}{l}\text { Quedas de idosos institucio- } \\
\text { nalizados: ocorrência e fatores } \\
\text { associados }\end{array}$ & $\begin{array}{l}40 \% \text { dos idosos relataram quedas nos últimos seis meses, e os fatores de risco } \\
\text { considerados significativos foram: sexo feminino, uso de medicamentos, visão de- } \\
\text { ficiente, ausência de atividade física, presença de osteoartrose, depressão, déficit } \\
\text { de força de preensão palmar e distúrbios no equilíbrio e na marcha. }\end{array}$ \\
\hline $\begin{array}{l}\text { Valcarenghi } \\
\text { et al. }\end{array}$ & $\begin{array}{l}\text { Alterações na funcionalidade/ } \\
\text { cognição e depressão em ido- } \\
\text { sos institucionalizados que so- } \\
\text { freram quedas }\end{array}$ & $\begin{array}{l}\text { Verificou-se que a maioria dos participantes eram mulheres e tinham baixa esco- } \\
\text { laridade; o risco de quedas aumentou com a idade; e idosos com menor tempo de } \\
\text { institucionalização caíram mais. Identificou-se a influência do uso de medicamen- } \\
\text { tos nas quedas. Os idosos que caíram apresentaram independência para as AVDs; } \\
\text { dos } 20 \text { idosos com escore sugestivo para déficit cognitivo, } 10 \text { sofreram quedas. } \\
\text { Não houve influência significativa entre depressão e quedas nos investigados. }\end{array}$ \\
\hline $\begin{array}{l}\text { Valcarenghi } \\
\text { et al. }\end{array}$ & $\begin{array}{l}\text { Ações institucionais alicerça- } \\
\text { das em diagnósticos de en- } \\
\text { fermagem para prevenção de } \\
\text { quedas em idosos }\end{array}$ & $\begin{array}{l}\text { Foram elaboradas propostas de ações institucionais relacionadas a receptibilidade } \\
\text { ao idoso pela instituição, adaptação do ambiente, treinamento físico dos idosos, } \\
\text { investigação e ensino de equipamentos auxiliares, promoção da independência, } \\
\text { avaliação das condições clínicas e custo benefício de tratamento medicamentoso. }\end{array}$ \\
\hline $\begin{array}{l}\text { Reis, Rocha } \\
\text { e Duarte }\end{array}$ & $\begin{array}{l}\text { Quedas: risco e fatores asso- } \\
\text { ciados em idosos instituciona- } \\
\text { lizados }\end{array}$ & $\begin{array}{l}62,9 \% \text { dos idosos participantes foram vítimas de quedas; as causas de maior distribui- } \\
\text { ção foram: dificuldade de caminhar, alteração de equilíbrio e tontura/vertigem, todos com, } \\
\text { respectivamente, } 16,1 \% \text {. Em relação ao risco, no teste de TUG, a queda foi classificada } \\
\text { com baixo risco; na escala de Berg, houve maior frequência }(61,3 \%) \text { de risco de quedas. }\end{array}$ \\
\hline $\begin{array}{l}\text { Rocha e } \\
\text { Duarte }\end{array}$ & $\begin{array}{l}\text { Prevalência do risco de quedas } \\
\text { em idosos de uma instituição } \\
\text { de longa permanência de San- } \\
\text { ta Maria (RS) }\end{array}$ & $\begin{array}{l}\text { Foi identificada alta prevalência do risco de quedas, principalmente em idosas, } \\
\text { quando comparadas aos idosos homens. }\end{array}$ \\
\hline $\begin{array}{l}\text { Uchida e } \\
\text { Borges }\end{array}$ & $\begin{array}{l}\text { Quedas em idosos institucio- } \\
\text { nalizados }\end{array}$ & $\begin{array}{l}\text { Observou-se que a frequência de quedas na população do estudo foi de apenas seis } \\
\text { idosos, que caíram nos últimos doze meses }(22,2 \%) \text {, totalizando nove quedas. No en- } \\
\text { tanto, dezesseis }(59,3 \%) \text { relataram algum medo de cair. Todos os idosos participantes } \\
\text { do estudo apresentaram de quatro a onze fatores de risco para quedas, sendo a presen- } \\
\text { ça de doença crônica, a polifarmácia, o uso de medicamento psicotrópico e o déficit cog- } \\
\text { nitivo os fatores de risco modificáveis mais frequentes. Já a idade elevada ( }>75 \text { anos) } \\
\text { e o sexo feminino foram os fatores que mais se destacaram entre os não modificáveis. }\end{array}$ \\
\hline $\begin{array}{l}\text { Álvares, } \\
\text { Lima e Silva }\end{array}$ & $\begin{array}{l}\text { Ocorrência de quedas em ido- } \\
\text { sos residentes em instituições } \\
\text { de longa permanência em Pe- } \\
\text { lotas, Rio Grande do Sul, Brasil }\end{array}$ & $\begin{array}{l}\text { Houve diferença entre os escores da escala de Berg nas populações estudadas } \\
(p<0,0001) \text {. Os resultados mostraram que a população idosa institucionalizada } \\
\text { apresenta nove vezes mais risco de cair do que idosos não institucionalizados ( } p \\
=0,0001 \text { ). }\end{array}$ \\
\hline $\begin{array}{l}\text { Menezes e } \\
\text { Bachion }\end{array}$ & $\begin{array}{l}\text { Ocorrência de quedas e seu } \\
\text { contexto num seguimento de } \\
\text { dois anos em idosos institucio- } \\
\text { nalizados }\end{array}$ & $\begin{array}{l}\text { O estudo apresentou } 54,2 \% \text { de prevalência de quedas. As quedas ocorreram, } \\
\text { predominantemente, durante o dia, fora do ambiente interno institucional, durante } \\
\text { a deambulação, em piso de cimento, seco, a partir de tropeços em algo, quando } \\
\text { estavam calçando chinelos. }\end{array}$ \\
\hline
\end{tabular}

Fonte: elaboração dos autores. 


\section{Principais resultados encontrados quanto às consequências das quedas de idosos residentes em Ilpis}

No que diz respeito aos episódios de queda, Uchida e Borges (2013) evidenciam que a maioria dos idosos apresenta algum medo de cair, mesmo quando não tem histórico de queda, o que favorece uma maior dependência na realização de atividades da vida diária e, consequentemente, aumenta o risco de quedas.

Silva et al. (2013) ressaltam que um idoso que sofreu uma queda pode desenvolver a "síndrome pós-queda", na qual o impacto psicológico pode reduzir as atividades básicas da vida diária proporcionalmente à grandeza das lesões resultantes, na tentativa de evitar novos episódios.

A possibilidade de queda em idosos gera preocupações e custos social e econômico elevados, decorrente das lesões provocadas, e afeta o convívio e a interação dessa população, causando medo no cumprimento de tarefas mais sofisticadas, como subir escadas, ou de atividades antes consideradas simples, como tomar banho, que passam a apresentar maior grau de dificuldade em decorrência da senilidade (GONÇALVES et al., 2014).

Quadro 5 - Principais resultados encontrados na categoria consequências das quedas em idosos residentes em Ilpis

\begin{tabular}{|l|l|l|}
\hline \multicolumn{1}{|c|}{ Autores } & \multicolumn{1}{|c|}{ Título } & \multicolumn{1}{c|}{ Principais resultados } \\
\hline Uchida e Borges & $\begin{array}{l}\text { Quedas em idosos institucio- } \\
\text { nalizados }\end{array}$ & $\begin{array}{l}\text { Observou-se que a frequência de quedas na população estudada foi } \\
\text { de apenas seis idosos, que caíram nos últimos doze meses (22,2\%), } \\
\text { totalizando nove quedas. No entanto, dezesseis participantes (59,3\%) } \\
\text { relataram algum medo de cair. Todos os idosos do estudo apresenta- } \\
\text { ram de quatro a onze fatores de risco para quedas, sendo a presença } \\
\text { de doença crônica, a polifarmácia, o uso de medicamento psicotrópico } \\
\text { e o déficit cognitivo os fatores de risco modificáveis mais frequentes. } \\
\text { Já a idade elevada (> 75 anos) e o sexo feminino foram os fatores que } \\
\text { mais se destacaram entre os não modificáveis. }\end{array}$ \\
\hline Silva et al. & $\begin{array}{l}\text { Correlação entre o risco de } \\
\text { queda e autonomia funcional } \\
\text { em idosos institucionalizados }\end{array}$ & $\begin{array}{l}\text { Dos participantes do estudo, 47\% apresentaram maior risco de queda, } \\
\text { e 53\%, menor risco. Ser do sexo feminino aumenta 3,9 vezes as chan- } \\
\text { ces de queda em relação ao sexo masculino. }\end{array}$ \\
\hline Gonçalves et al. & $\begin{array}{l}\text { Avaliação das funções cogniti- } \\
\text { vas, qualidade do sono, tempo } \\
\text { de reação e risco de quedas } \\
\text { em idosos institucionalizados }\end{array}$ & $\begin{array}{l}\text { A preocupação com a possibilidade de quedas realizando atividades } \\
\text { cotidianas em geral, avaliada por meio da EEQ, apresentou média de } \\
\text { 31,75 pontos, o que revela medo de cair. Acredita-se que possa haver } \\
\text { relação entre a preocupação com a possibilidade de sofrer quedas e } \\
\text { o declínio das atividades de interação social, de prática de atividades } \\
\text { físicas e de vida diária. }\end{array}$ \\
\hline
\end{tabular}

Fonte: elaboração dos autores.

\section{Conclusão}

Em idosos institucionalizados, o risco de quedas ocorre devido à vulnerabilidade desses idosos em desenvolver um perfil clinicofuncional e psicocognitivo que se associa aos fatores de risco de queda, sejam intrínsecos - idade, sexo, alterações hormonais, crenças, etc. - ou extrínsecos - nutrição, comorbidades, interação medicamentosa, episódios de quedas, etc. -, resultando em instabilidade corporal causada por diversas alterações estruturais. 


\section{Falls of risk assessment of elderly residents in long stay Brazilian institutions: an integrative review}

\section{Abstract}

Falls among the elderly are a major clinical problem and public health because of its high incidence, complications and medical care costs. In this perspective, the present study was was to analyze the risk assessment of falls in elderly people living in a long-term institution, based on an integrative review. We analyzed registered articles in SciElo and Medicine ${ }^{\circledR}$ research bases; the period 2010 to 2015; English and language resulting from the crossing of the following descriptors: long-term care facility, elderly, and risk of falls, using the indicator Boolean AND. Among the 19 items used was evident institutionalization, female, acimas age 70 years, presence of comorbidities, medication use and polypharmacy, local and most common period as decisive to make the elderly vulnerable to episodes of fall.

Keywords: Evaluation. Elderly. Fall.

\section{Referências}

ALMEIDA, Patrícia; NEVES, Rui. As quedas e o medo de cair em pessoas idosas institucionalizadas. Kairós Gerontologia, São Paulo, v. 15, n. 3, p. 27-43, set. 2012 .

ÁLVARES, Liege Mata; LIMA, Rosângela da Costa; SILVA, Ricardo Azevedo. Ocorrência de quedas em idosos residentes em instituições de longa permanência em Pelotas, Rio Grande do Sul, Brasil. Cadernos de Saúde Pública, Rio de Janeiro, v. 26, n. 1, p. 31-40, 2010.

ALVES, Natália Beghine; SCHEICHER, Marcos Eduardo. Equilíbrio postural e risco para queda em idosos da cidade de
Garça, SP. Revista Brasileira de Geriatria e Gerontologia, Rio de Janeiro, v. 14, n. 4, p. 763-768, 2011.

ANTES, Danielle Ledur et al. Medo de queda recorrente e fatores associados em idosos de Florianópolis, Santa Catarina, Brasil. Cadernos de Saúde Pública, Rio de Janeiro, v. 29, n. 4, p. 758-768, 2013.

BORGES, Cíntia Lira et al. Avaliação da fragilidade de idosos institucionalizados. Acta Paulista de Enfermagem, São Paulo, v. 26, n. 4, p. 318-22, 2013.

BUKSMAN, S. et al. Queda em Idosos: prevenção. Projeto Diretrizes. Brasília: Associação Médica Brasileira e Conselho Federal de Medicina, 2008.

CAMARANO, Ana Amélia; KANSO, Solange. As instituições de longa permanência para idosos no Brasil. Revista Brasileira de Estudos de População, Rio de Janeiro, v. 27, n. 1, p. 232-235, jan./jun. 2010.

DE SOUZA TEIXEIRA, Camila et al. Prevalência do risco de quedas em idosos de uma instituição de longa permanência de Santa Maria (RS). Kairós Gerontologia, São Paulo, v. 17, n. 1, p. 45-56, 2014.

DEL DUCA, Giovâni Firpo; ANTES, Danielle Ledur; HALLAL, Pedro Curi. Quedas e fraturas entre residentes de instituições de longa permanência para idosos. Revista Brasileira de Epidemiologia, São Paulo, v. 16, n. 1, p. 68-76, 2013.

DOS SANTOS LOPES, Mariane Negrão Serra; PASSERINI, Cintia Gomes; TRAVENSOLO, Cristiane de Fátima. Eficácia de um protocolo fisioterapêutico para equilíbrio em idosos institucionalizados. Semina: Ciências Biológicas e da Saúde, Londrina, v. 31, n. 2, p. 143-152, 2010.

FECHINE, Basílio Rommel Almeida; TROMPIERI, Nicolino. O processo de envelhecimento: as principais alterações que acontecem com o idoso com o passar dos anos. InterSciencePlace, Campos dos Goytacazes (RJ), v. 20, n. 1, p. 107-194, jan./mar. 2015. 
FERREIRA, Denise Cristina de Oliveira; YOSHITOME, Aparecida Yoshie. Prevalência e caraterísticas das quedas de idosos institucionalizados. Revista Brasileira de Enfermagem, Brasília, v. 63, n. 6, p. 991-997, nov./dez. 2010.

FERREIRA, Lucas Lima et al. Risco de queda em idosos com doença de Alzheimer institucionalizados. ConScientia e Saúde, v. 12, n. 3, p. 379-385, 2013a.

FERREIRA, Lucas Lima et al. Risco de queda em idosos institucionalizados com doença de Alzheimer. Kairós Gerontologia, São Paulo, v. 16 , n. 3, p. 95-105, $2013 \mathrm{~b}$.

GONÇALVES, Daianne et al. Avaliação das funções cognitivas, qualidade de sono, tempo de reação e risco de quedas em idosos institucionalizados. Estudos Interdisciplinares sobre o Envelhecimento, Porto Alegre, v. 19, n. 1, p. 96-108, 2014.

INSTITUTO BRASILEIRO DE GEOGRAFIA E ESTATÍSTICA (IBGE). Projeções e estimativas da população do Brasil e das unidades da Federação. Rio de Janeiro, 2016. Disponível em: <http://www.ibge.gov.br/apps/ populacao/projecao/index.html >. Acesso em: 20 mar. 2017.

LOJUDICE, Daniela Cristina et al. Quedas de idosos institucionalizados: ocorrência e fatores associados. Revista Brasileira de Geriatria e Gerontologia, Rio de Janeiro, v. 13, n. 3, p. 403-412, 2010.

MENEZES, Ruth Losada de; BACHION, Maria Márcia. Ocorrência de quedas e seu contexto num seguimento de dois anos em idosos institucionalizados. Revista Eletrônica de Enfermagem, Goiânia, v. 14, n. 3, p. 550558 , jul./set. 2012a.

MENEZES, Ruth Losada de; BACHION, Maria Márcia. Condições visuais autorrelatadas e quedas em idosos institucionalizados. Revista Brasileira de Oftamologia, Rio de Janeiro, v. 71, n. 1, p. 23-27, 2012b.
ORGANIZAÇÃO MUNDIAL DA SAÚDE (OMS). Resumo: Relatório Mundial de Envelhecimento e Saúde. Genebra: OMS, 2015. Disponível em: <http://sbgg.org.br/ wp-content/uploads/2015/10/OMS-ENVELHECIMENTO-2015-port.pdf>. Acesso em: 10 mar. 2016.

REIS, Luciana Araújo dos; ROCHA, Thais de Souza; DUARTE, Stênio Fernando Pimentel. Quedas: risco e fatores associados em idosos institucionalizados. Revista Baiana de Enfermagem, Salvador, v. 28, n. 3, p. 225-234, set./dez. 2014.

ROMAN, Arlete Regina; FRIEDLANDER, Maria Romana. Revisão integrativa de pesquisa aplicada à enfermagem. Cogitare Enfermagem, Curitiba, v. 3, n. 2, p. 109-112, jul./dez. 1998.

SILVA, José Mário Nunes da et al. Correlação entre o risco de queda e autonomia funcional em idosos institucionalizados. Revista Brasileira de Geriatria e Gerontologia, Rio de Janeiro, v. 16, n. 2, p. 337-346, 2013.

SOUZA, Marina Celly Martins Ribeiro de et al. Perfil de idosas que sofreram quedas em uma instituição de longa permanência. $R e$ com - Revista de Enfermagem do Centro-Oeste Mineiro, Divinópolis (MG), v. 2, n. 2, p. 220-227, maio/ago. 2012.

UCHIDA, Julia Emiko Fleming; BORGES, Sheila de Melo. Quedas em idosos institucionalizados. Kairós Gerontologia, São Paulo, v. 16, n. 3, p. 83-94, 2013.

VALCARENGHI, Rafaela Vivian et al. Alterações na funcionalidade/cognição e depressão em idosos institucionalizados que sofreram quedas. Acta Paulista de Enfermagem, São Paulo, v. 24, n. 6, p. 828-33, 2011.

VALCARENGHI, Rafaela Vivian et al. Ações institucionais alicerçadas em diagnósticos de enfermagem para prevenção de quedas em idosos. Rev Rene: Revista da Rede de Enfermagem do Nordeste, v. 15, n. 2, 2014. 